\title{
Non-communicable disease mortality rates using the verbal autopsy in a cohort of middle aged and older populations in Beirut during wartime, 1983-93
}

\author{
A M Sibai, A Fletcher, M Hills, O Campbell
}

American University of Beirut, Faculty of Health Sciences, Department of Epidemiology and Biostatistics, Beirut, Lebanon

A M Sibai

Epidemiology Unit, Department of Epidemiology and Population Health, London School of Hygiene and Tropical Medicine, London A Fletcher

Medical Statistics Unit, Department of Epidemiology and Population Health, London School of Hygiene and Tropical Medicine

$M$ Hills

Maternal and Child Epidemiology Unit, Department of Epidemiology and Population Health, London School of Hygiene and Tropical Medicine

O Campbell

Correspondence to: Dr Sibai

(ansibai@aub.edu.lb)

Accepted for publication 27 October 2000
Abstract

Study objectives-Health priorities in middle to low income countries, such as Lebanon, have traditionally been assumed to follow those of a "typical" developing country, with a focus on the young and on communicable diseases. This study was carried out to quantify the magnitude of communicable and non-communicable disease mortality and to examine mortality pattern among middle aged and older populations in an urban setting in Lebanon.

Design and participants-A representative cohort of 1567 men and women ( $\geqslant 50$ years) who had participated in a cross sectional multi-dimensional health survey in Beirut, Lebanon in 1983 and were followed up 10 years later. Vital status was ascertained and causes of death were obtained through verbal autopsy.

Results-Total mortality rates were estimated at 33.7 and 25.2/1000 person years among men and women respectively. In both sexes, the leading causes of death were non-communicable, mainly circulatory diseases $(60 \%)$ and cancer $(15 \%)$. For all cause mortality, men had significantly higher risk than women (age adjusted rate ratio, $R R=1.42,95 \%$ confidence intervals $(C I)=1.16,1.72)$ especially at younger ages. Except for cerebrovascular diseases, renal problems and injuries attributable to falls and fractures, men were also at higher cause specific mortality risk than women, in particular, for ischaemic heart disease $(R R=2.24,95 \% \mathrm{CI}=1.62,3.12)$. Comparison with earlier death certificate data in Lebanon and current estimates from other regions in the world showed the magnitude of cardiovascular disease over time.

Conclusions-The results from this first cohort study in the Arab region show, in contrast with popular perception, a mortality pattern more like a developed country than a developing one. Strategies of public health activities, in particular for countries in transition, need to be continuously re-assessed in light of empirical epidemiological data and other health indicators for evidence-based decision making.

(F Epidemiol Community Health 2001;55:271-276)
There is increasing awareness that most developing countries are experiencing the epidemiological transition in which causes of death shift from primarily infectious to noncommunicable diseases such as cardiovascular disease (CVD) and cancer. ${ }^{1-3}$ The increase in the relative burden of non-communicable diseases places greater demands on the health, social and economic care systems of countries already faced with limited resources and where communicable and maternal causes of ill health remain important public health issues. ${ }^{4}$ Efforts are now being made to quantify the global burden of disease to facilitate rational decision making. ${ }^{5}$ However, the lack of appropriate denominators and adequate sources of data to appraise the increasing importance of CVD means that donors and health planners in less developed countries often fall back on the assumption that resources should focus on the young and on infectious diseases.

Lebanon is a small (total population around 3.5 million) middle income country that recently emerged from 16 years of civil wars, foreign invasions and intrusions (1975-1991). In Lebanon, there are considerable gaps in the current epidemiological knowledge for most diseases. The most recent census was conducted in 1932 when Lebanon was still a French mandate. Since independence in 1943, and for various political reasons, efforts to collect demographic data and population statistics have not been welcome. Vital registration is unreliable while government statistical surveys and health statistics were, until very recently, non-existent. Reliable information on causes of death is not available, in particular, among the older age groups. A "Population and Housing" survey conducted by the Ministry of Social Affairs estimated the crude death rate for 1996 as 7.4 per 1000 population. ${ }^{6}$ While CVD and cancers accounted for around $31 \%$ and $10 \%$ of deaths reported in the year before the survey respectively, ill defined conditions represented a further $43 \%$ in the total sample, and $51 \%$ among those aged 60 years and older.

Given this background, this follow up study was undertaken to describe the level and causes of mortality in a cohort of middle aged and older populations in an urban setting in Lebanon. In the absence of other more reliable sources of mortality data, the study used verbal interviews for assessment of vital status and causes of death. Comparisons are made with data from developed and developing countries and with earlier data from Lebanon to contribute to our 
knowledge of the relative magnitude of disease burden over time.

\section{Methods}

In 1983, the Faculty of Health Sciences of the American University of Beirut conducted a multidimensional community-based health interview survey in Beirut, the largest city (around $13 \%$ of the population) and the capital of Lebanon. The objective of the 1983 survey was to establish a Population Laboratory to identify health needs at the community level, for planning and resource allocation. In the absence of a population or housing census that could provide a sampling frame, the 1983 sample was obtained using a two stage area design based on the geographical division of Beirut into zones and sectors as used by the Lebanese Electricity Company. Full details of sampling procedures are available elsewhere. ${ }^{7}$ The sampling approach was made to ensure a sufficiently representative sample of the residents of the city of Beirut.

Between June 1993 and August 1994, men and women aged 50 years and over in the Beirut 1983 Survey $(n=1786)$ were retrospectively traced for assessment of vital status. Data on causes of death were obtained using verbal interviews. The interview schedule, verbal autopsy, was based, with modifications, on that used in a similar follow up study of a cohort of older adults in Brazil. ${ }^{8}$ In this study, causes of death based on the verbal autopsy largely agreed with death certificates and hospital notes. ${ }^{8}$ The interview schedule included three main sections: (1) details on the year and age at death, country and place of death, identity and speciality of the attending physician; (2) circumstances of death elicited from the informant using open-ended questions as to the symptoms, signs and complaints around the time of death; and (3) a detailed medical history, age at onset, a checklist of morbid conditions suffered by the deceased one year before death, contacts with health services and accidents. The completed forms were reviewed by a physician to assign the underlying cause of death using the International Classification of Diseases, Injuries, and Causes of Death, 9th revision (ICD-9). In the case when there were ambiguities, a different interviewer was sent to the field for a re-interview and information recorded was evaluated by two physicians for a consensus.

The total observation time for each subject was calculated in person years divided into age and period bands within which the rate for the subject was considered to be constant. This was done using the Lexis command ${ }^{9}$ of statistical package STATA. ${ }^{10}$ Incidence density rates and associated confidence intervals (CI) were calculated per 1000 person years at risk with follow up time beginning at the time of the Beirut 1983 survey until censoring. Age adjusted Mantel-Haenzsel sex rate ratios (RR) and their $95 \%$ CI for each cause of death were also calculated.

\section{Results}

FOLLOW UP RESULTS AND MORTALITY OUTCOME Out of the sample of 1786 study subjects aged 50 years and over in 1983, a total of 1567 subjects $(87.7 \%)$ were successfully traced in 1993/94 (fig 1). Of these, 1151 were alive and 416 dead. The mean time to death was five years (SD three years). For the remaining 219 respondents, the vital status and, with less certainty, the year at censoring could be elicited for $140(63.9 \%)$ subjects, of whom 94 were alive and 46 dead. However, because they refused to participate or because they had moved and/or an appropriate informant could not be identified, data on causes of death could not be assessed. Follow up interviews of the 1567 traced subjects were conducted at the same address as the 1983 Survey in $84.3 \%$ of cases. The other interviews were conducted either in a newly identified address $(11.7 \%)$ or by phone contact $(3.9 \%)$.

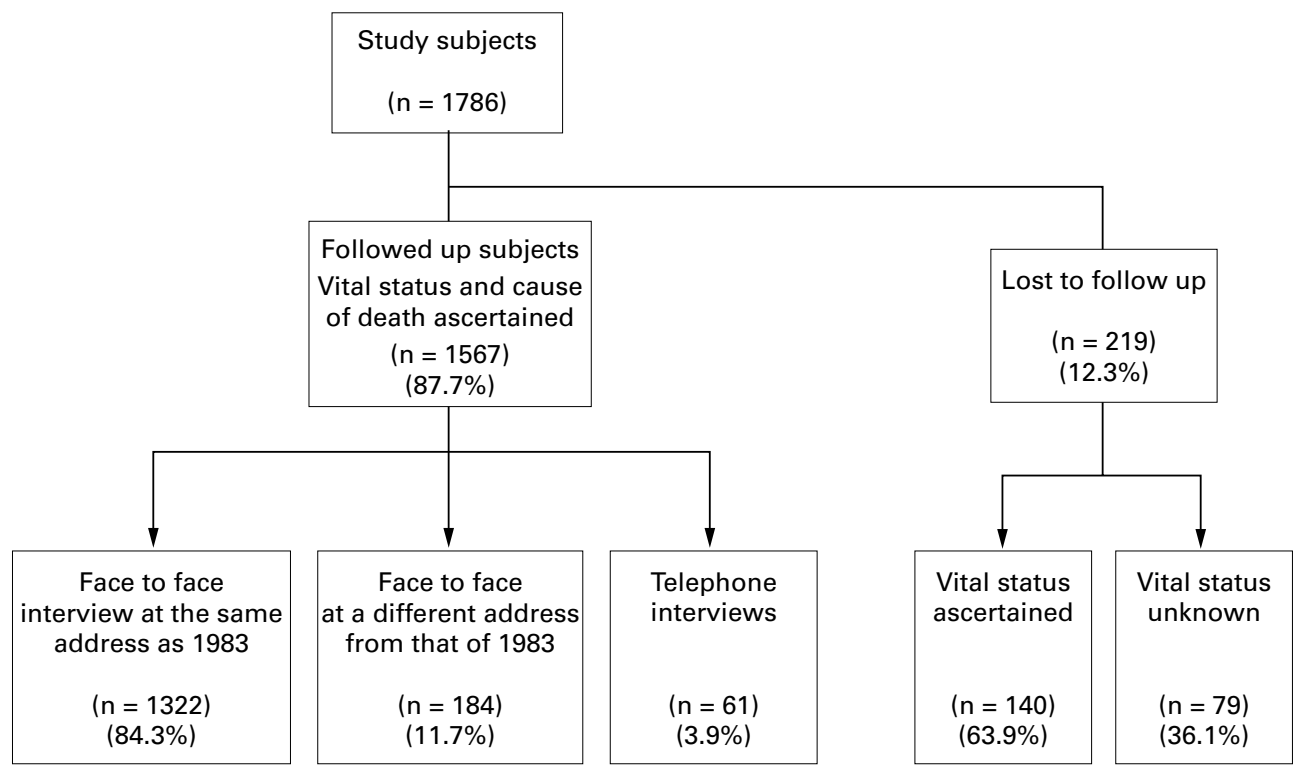

Figure 1 Follow up results in the field, Beirut 1983-1993/4. 
Table 1 Distribution of underlying causes of death according to major categories of diseases (ICD-9) by sex and age at death among people aged 50 years and above at start of follow up, Beirut 1983-1993/4

\begin{tabular}{|c|c|c|c|c|c|c|c|c|c|c|c|c|c|c|c|}
\hline & & \multicolumn{6}{|l|}{ Men } & \multicolumn{6}{|l|}{ Women } & \multicolumn{2}{|l|}{ Total } \\
\hline & & \multicolumn{6}{|c|}{ Age groups } & \multicolumn{6}{|c|}{ Age groups } & \multirow[b]{3}{*}{ Number } & \multirow[b]{3}{*}{$\%$} \\
\hline & & $50-64$ & & $65-79$ & & $80+$ & & $50-64$ & & $65-79$ & & $80+$ & & & \\
\hline \multicolumn{2}{|c|}{ Cause of death } & Number & $\%$ & Number & $\%$ & Number & $\%$ & Number & $\%$ & Number & $\%$ & Number & $\%$ & & \\
\hline I & Communicable diseases & 0 & 0.0 & 5 & 4.6 & 3 & 4.7 & 0 & 0.0 & 0 & 0.0 & 1 & 1.4 & 9 & 2.2 \\
\hline \multirow[t]{12}{*}{ II } & Non-communicable diseases & & & & & & & & & & & & & & \\
\hline & Cancer & 15 & 23.8 & 20 & 18.3 & 2 & 3.1 & 10 & 28.6 & 11 & 15.5 & 4 & 5.5 & 62 & 14.9 \\
\hline & Diabetes & 1 & 1.6 & 9 & 8.2 & 1 & 1.6 & 1 & 2.9 & 2 & 2.8 & 1 & 1.4 & 15 & 3.6 \\
\hline & Circulatory diseases (sub-total) & 38 & 60.3 & 63 & 57.2 & 46 & 71.9 & 19 & 54.3 & 43 & 60.6 & 40 & 54.8 & 249 & 59.8 \\
\hline & Ischaemic heart disease & 30 & 47.6 & 55 & 50.0 & 29 & 45.3 & 9 & 25.7 & 21 & 29.6 & 24 & 32.9 & 168 & 40.4 \\
\hline & Cerebrovascular diseases & 8 & 12.7 & 4 & 3.6 & 8 & 12.5 & 9 & 25.7 & 13 & 18.3 & 11 & 15.1 & 53 & 12.7 \\
\hline & Other heart/circulatory & & & & & & & & & & & & & & \\
\hline & diseases & 0 & 0.0 & 4 & 3.6 & 9 & 14.1 & 1 & 2.9 & 9 & 12.7 & 5 & 6.8 & 28 & 6.7 \\
\hline & Respiratory diseases & 1 & 1.6 & 3 & 2.7 & 2 & 3.1 & 1 & 2.9 & 2 & 2.8 & 0 & 0.0 & 9 & 2.2 \\
\hline & Digestive diseases & 1 & 1.6 & 3 & 2.7 & 0 & 0.0 & 0 & 0.0 & 0 & 0.0 & 0 & 0.0 & 4 & 1.0 \\
\hline & Renal problems & 2 & 3.2 & 1 & 0.9 & 1 & 1.6 & 3 & 8.6 & 4 & 5.6 & 2 & 2.7 & 13 & 3.1 \\
\hline & Senile and ill defined & 0 & 0.0 & 1 & 0.9 & 4 & 6.3 & 0 & 0.0 & 8 & 11.3 & 16 & 21.9 & 29 & 7.0 \\
\hline \multirow[t]{5}{*}{ III } & Injuries & & & & & & & & & & & & & & \\
\hline & Motor vehicle accidents & 1 & 1.6 & 2 & 1.8 & 0 & 0.0 & 1 & 2.9 & 0 & 0.0 & 0 & 0.0 & 4 & 1.0 \\
\hline & Falls and fractures & 0 & 0.0 & 1 & 0.9 & 4 & 6.3 & 0 & 0.0 & 0 & 0.0 & 9 & 12.3 & 14 & 3.4 \\
\hline & War related injuries & 4 & 6.3 & 2 & 1.8 & 1 & 1.6 & 0 & 0.0 & 1 & 1.4 & 0 & 0.0 & 8 & 1.9 \\
\hline & Total & 63 & 100 & 110 & 100 & 64 & 100 & 35 & 100 & 71 & 100 & 73 & 100 & 416 & 100 \\
\hline
\end{tabular}

CAUSE OF DEATH STRUCTURE BY AGE AND SEX The distribution of the underlying causes of death by sex and age at death is provided in table 1. For comparative purposes, causes of death were grouped under three main headings according to the framework used by Murray and Lopez Global Burden of Disease (GBD) study: communicable diseases, noncommunicable diseases and injuries. ${ }^{5}$ During the 10 year follow up period, 237 deaths occurred among men and 179 among women. Their median age at death was 71 (range 53-101 years) and 76 (range 53-99 years) respectively. The most important causes were non-communicable diseases, mainly circulatory disease $(\mathrm{n}=249(60 \%)$; ICD-9 codes $390-459)$ and cancer ( $n=62(15 \%)$; codes 140-208). Among circulatory diseases, ischaemic heart disease (codes 410-414) accounted for the majority of the mortality burden ( $\mathrm{n}=$ $168,68 \%$ ) followed by cerebrovascular diseases $(n=53(21 \%)$; codes 430-438).

Around $6 \%$ of total deaths were caused by injuries, both intentional and unintentional. Overall, injuries were concentrated among men in the younger age groups, although the pattern varied by type of injury. While the majority of motor vehicle accidents occurred among men in the younger age groups, fractures, mainly of the hip and pelvis, concentrated among women above 80 years.

MORTALITY RATES BY AGE AND SEX

A graphical representation of trends in all cause and CVD mortality per 1000 person years, by age (on a log scale), is presented in figure 2 . Mortality rates increased consistently with age and were higher in men than women, especially for the younger age groups. Except for cerebrovascular diseases, renal problems, injuries attributable to falls and fractures, and ill defined diseases, men were at higher mortality risk than women (table 2). Men exhibited a significantly higher mortality risk for deaths attributable to communicable diseases (age adjusted $\mathrm{RR}=8.83)$, non-communicable diseases $(R R=1.36)$, in particular circulatory diseases $(R R=1.54)$ and ischaemic heart disease $(\mathrm{RR}=2.24)$.

Table 2 Ten year cause specific mortality rates, rate ratios by sex and their 95\% confidence intervals (CI), among people aged 50 years and above at start of follow up, Beirut 1983-1993/4

\begin{tabular}{|c|c|c|c|c|c|c|c|c|c|}
\hline & & \multicolumn{3}{|c|}{$\operatorname{Men}(M)$} & \multicolumn{3}{|c|}{ Women (W) } & \multicolumn{2}{|l|}{$M / W$} \\
\hline \multicolumn{2}{|c|}{ Cause of death } & Number & Rate* $^{*}$ & $95 \% C I$ & Number & Rate $^{*}$ & $95 \% C I$ & Rate ratiof & $95 \% C I$ \\
\hline I & Communicable diseases & 8 & 1.14 & $0.57,2.27$ & 1 & 0.14 & $0.02,1.00$ & 8.83 & $1.13,68.8$ \\
\hline \multirow[t]{11}{*}{ II } & Non-communicable diseases & 214 & 30.43 & $26.6,34.8$ & 167 & 23.50 & $20.2,27.4$ & 1.36 & $1.11,1.68$ \\
\hline & Cancer & 37 & 5.26 & $3.81,7.26$ & 25 & 3.52 & $2.38,5.21$ & 1.49 & $0.89,2.49$ \\
\hline & Diabetes & 11 & 1.56 & $0.87,2.82$ & 4 & 0.56 & $0.21,1.50$ & 2.83 & $0.89,8.84$ \\
\hline & Circulatory diseases & 147 & 20.90 & $17.8,24.6$ & 102 & 14.36 & $11.8,17.4$ & 1.54 & $1.19,1.98$ \\
\hline & Ischaemic heart disease & 114 & 16.21 & $13.5,19.5$ & 54 & 7.60 & $5.82,9.93$ & 2.24 & $1.62,3.12$ \\
\hline & Cerebrovascular disease & 20 & 2.84 & $1.84,4.41$ & 33 & 4.64 & $3.30,6.53$ & 0.65 & $0.37,1.12$ \\
\hline & Other heart diseases & 13 & 1.85 & $1.07,3.18$ & 15 & 2.11 & $1.27,3.50$ & 0.98 & $0.47,2.03$ \\
\hline & Respiratory diseases & 6 & 0.85 & $0.98,1.89$ & 3 & 0.42 & $0.14,1.31$ & 2.04 & $0.51,8.19$ \\
\hline & Digestive diseases & 4 & 0.57 & $0.21,1.52$ & 0 & 0 & - & - & - \\
\hline & Renal problems & 4 & 0.57 & $0.21,1.52$ & 9 & 1.27 & $0.66,2.44$ & 0.45 & $0.14,1.47$ \\
\hline & Senile and ill defined & 5 & 0.71 & $0.29,1.71$ & 24 & 3.38 & $2.26,5.04$ & 0.26 & $0.10,0.68$ \\
\hline \multirow[t]{5}{*}{ III } & Injuries & 15 & 2.13 & $1.29,3.54$ & 11 & 1.55 & $0.86,2.79$ & 1.52 & $0.70,3.31$ \\
\hline & Motor vehicle accidents & 3 & 0.43 & $0.14,1.32$ & 1 & 0.14 & $0.02,1.00$ & 3.08 & $0.32,29.6$ \\
\hline & Falls and fractures & 5 & 0.71 & $0.29,1.71$ & 9 & 1.27 & $0.66,2.44$ & 0.68 & $0.24,1.94$ \\
\hline & War related injuries & 7 & 1.00 & $0.47,2.09$ & 1 & 0.14 & $0.02,1.00$ & 7.12 & $0.87,58.4$ \\
\hline & Total & 237 & 33.70 & $29.67,38.27$ & 179 & 25.20 & $21.76,29.17$ & 1.42 & $1.16,1.72$ \\
\hline
\end{tabular}

^Rates per 1000 person years. †Age adjusted Mantel-Haenszel rate ratio comparing men with women. 

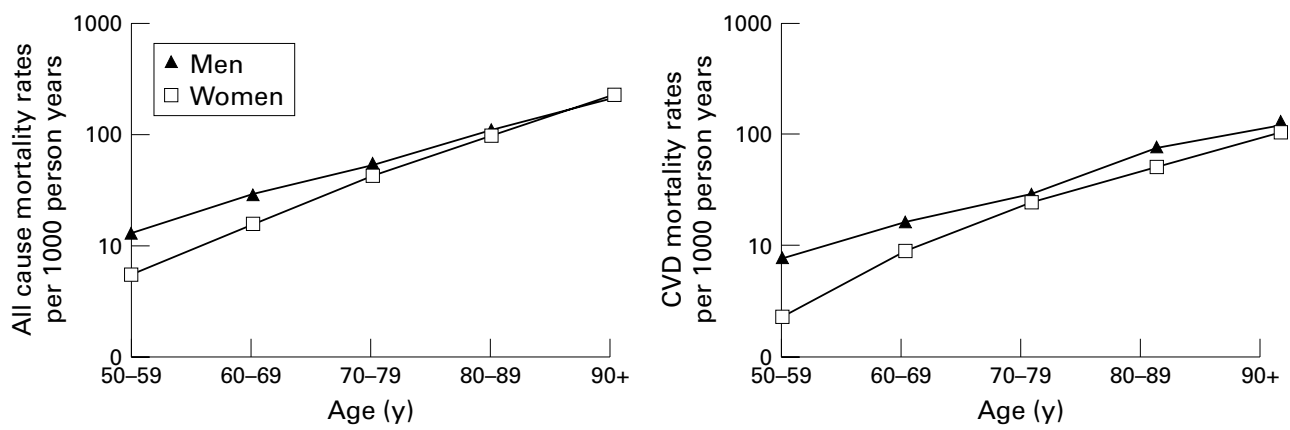

Figure 2 Ten year all cause and cardiovascular mortality rates (per 1000 person years) among men and women aged 50 years and over by age groups on a log scale, Beirut 1983-93/94.

\section{Discussion}

To date, cohort studies and population-based mortality data in the Arab region are largely lacking. The findings of this study show that non-communicable diseases, particularly CVD and cancer, are the leading causes of death among those aged 50 years and over in Beirut, accounting for around $60 \%$ and $15 \%$ of total deaths respectively. While methodological issues and sociocultural factors may have played a part in diagnosis and reporting, these cannot totally account for such high proportional mortality rates. Total mortality rates were significantly higher in men than women, and this was shown to vary by age. Controlling for age, men had around 1.4 times mortality risk than women. Much of the excess risk among men was attributable to ischaemic heart disease rather than cerebrovascular disease mortality, a finding that is consistent with other epidemiological studies. ${ }^{11}$ Furthermore, mortality rates from CVD reflect a pattern comparable to what is observed in industrialised countries such as the USA and England and Wales. ${ }^{12}$ The results for trends in mortality by age and gender are also consistent with reports from populations in the West. ${ }^{13}$

The utility of these findings, however, is largely dependent on the quality of mortality data. A number of factors may affect the reliability of verbal autopsies: the prevalence of the condition in question, the socioeconomic status of the informant and relationship to the decedent, seriousness of the illness and certainly age at death. ${ }^{14}$ In our study, more than half $(58 \%)$ of the subjects had been admitted to a hospital in the year before death, and around one third of the deaths occurred in a hospital setting. Consequently, the information obtained was likely to be shaped by contact with the physician attending to the patient during terminal illness. It is possible, however, that data on the circumstances of deaths that occurred early in the study period may have suffered from poorer recall. Another potential limitation lies in the closeness of the respondent to the decedent around the time of death. The majority of interviews $(80 \%)$ were conducted with a relative or a caregiver who had lived with the subject in the same household in the year preceding the death event. In another $12 \%$ of the cases, the informant had lived either in the same building or within a few blocks from the house the deceased. Reliability of our verbal interviews by informant characteristics
KEY POINTS

- In countries that lack reliable sources of mortality data, the utility of verbal autopsy can be viably extended to cohort studies for assessing causes of death.

- The lack of reliable health information and the traditional dichotomy between developed and developing countries mask heterogeneity within countries and may result in misleading appraisals of research and health priorities.

- Strategies of public health activities, in particular for countries in transition, need to be formulated with an understanding of disease burden within rather than between populations, and to be continuously re-assessed in light of empirical data for evidence-based decision making.

was examined at the outset of the study in a pilot sample of 30 deaths. Using broad categories of causes of mortality (communicable diseases, cancer, CVDs, other non-communicable diseases, injuries and ill defined conditions), the overall level of agreement was $90 \%$ ( $\kappa$ statistics $=0.85$ ) and this did not vary by respondent relationship to decedent. Furthermore, our use of a broad system of classification of mortality provided a balance between the need for details on specific causes of death against the inevitable lack of reliability inherent in a more detailed cause of death structure.

Assessment of the validity, on the other hand, of our verbal autopsies was hampered by the lack of other traditional sources of mortality data in the country. Hospital records were either inaccessible, incomplete or missing. Similarly, a search exercise at the Vital Registration Unit revealed that the cause of death was missing in $68 \%$ of death certificates and "old age and ill defined conditions" constituted a further $5 \%$ of all registered deaths. Countries that lack reliable sources of data are increasingly adopting surveys and lay reporting schemes to measure broad cause of death patterns. Verbal autopsies have been used in South Africa, ${ }^{15}$ India, ${ }^{16}$ and more recently in Jordan ${ }^{17}$ to assign causes of death among adults. In comparison with the gold standard (death certificates and hospital records), physician review of verbal autopsies had a high diagnostic accuracy for CVD (cause specific mortality fraction 
Table 3 Comparison of the distribution of deaths in Beirut based on death certificates (1966) and this study (1983-93) with estimates in developed, developing regions and the Middle Eastern Crescent based on the Global Burden of Disease Study in 1990

\begin{tabular}{|c|c|c|c|c|c|c|}
\hline \multicolumn{2}{|c|}{ Study } & Abou-Daoud study* & \multicolumn{3}{|c|}{ Global Burden of Disease study ${ }^{*}$} & This study \\
\hline \multirow{2}{*}{\multicolumn{2}{|c|}{$\begin{array}{l}\text { Year } \\
\text { Age }\end{array}$}} & 1966 & \multicolumn{3}{|l|}{1990} & 1983-93 \\
\hline & & All ages & \multicolumn{2}{|l|}{ All ages } & $>60$ years & $>60$ years \\
\hline \multicolumn{2}{|c|}{ Place } & Beirut/Lebanon & Developed regionst & Developing regionst & Middle Eastern Crescent & Beirut/Lebanon \\
\hline \multicolumn{2}{|c|}{ Number of deaths } & 2748 & $10883 \times 10^{3}$ & $39088 \times 10^{3}$ & $1376 \times 10^{3}$ & 378 \\
\hline I & Communicable diseases & 7.1 & 5.3 & 41.2 & 10.5 & 2.4 \\
\hline \multirow[t]{6}{*}{ II } & Non-communicable diseases & & & & & \\
\hline & Cancer & 11.1 & 22.3 & 9.5 & 14.3 & 14.5 \\
\hline & Cardiovascular diseases & 48.4 & 48.9 & 23.1 & 54.7 & 60.6 \\
\hline & Other causes & 21.2 & 15.4 & 17.0 & 18.3 & 9.0 \\
\hline & Senile and ill defined & 5.2 & - & - & - & 7.7 \\
\hline & Injuries & 7.0 & 7.4 & 8.7 & 2.6 & 5.8 \\
\hline
\end{tabular}

* Source: Abou-Daoud, $1967,{ }^{19}$ calculated from the annex in Murray and Lopez, $1997 .{ }^{20} \dagger$ Developed regions: Established market economies and former socialist countries, Developing regions: all others.

within $10-19 \%$ of the true value) and neoplasms $(<10 \%)$ in a sample of 796 adult deaths in Tanzania, Ethiopia and Ghana. ${ }^{18}$

While the nature of study subjects selected for this investigation from an urban setting limits our ability to generalise the study results to all the population, Lebanon remains largely an urbanised country (around $85 \%){ }^{6}$ To our knowledge, this study provides the first population-based data available in the Arab region quantifying incidence density mortality rates experienced in a geographically well defined population over a specific period of time. The study achieved a relatively high follow up rate for a population then undergoing a protracted war and only a low proportion of deaths was coded to "ill defined". Moreover, it focused attention on middle aged and older people, an often neglected sub-population in research and policy in the region.

In the absence of published data on vital statistics in Lebanon, it is difficult to examine cause specific mortality trends in recent years. The only study ever published, which examined causes of death before the war, was undertaken in 1966 and was based on death certificates and registers of the Municipal Health Department in the city of Beirut. ${ }^{19}$ Table 3 reproduces the distribution of deaths cited in this earlier study in Beirut and in the Global Burden of Disease (GBD) Study in 1990 across different regions, including the Middle Eastern Crescent. ${ }^{20}$ The comparison suggests that, in contrast with popular national and international perception, the epidemic of CVD has not just emerged; rather, its onset probably dates back to the 1960 s. In one of the few epidemiological studies of coronary heart disease conducted before the war, AbouDaoud starts his introduction with the statement: "Coronary heart disease seems to be the leading cause of death among Lebanese of the middle and older age groups". ${ }^{21}$ The relative stability of mortality trends over 30 years suggests that, although the war may not have dramatically affected our epidemiological transition, it had probably delayed any further developments. Baseline data from this cohort study revealed increased levels of chronic disease morbidity (hypertension, 25\%; and diabetes, $13 \%$ ) and a high prevalence of the major risk factors such as smoking (44\% among men and $24 \%$ among women). At the time when several countries, essentially through primary prevention, are showing marked decreases in CVD mortality from the $1960 \mathrm{~s},{ }^{122-24}$ health policies and practices in the country continue to favour curative and high-tech medical services.

The recent publication of the mortality results of the GBD study in $1997,{ }^{5}$ has called attention to the world's health transition and to the policy implications of such global comparisons. ${ }^{25}{ }^{26}$ Because of the great diversity in social, economic and health characteristics within groups and countries, all categorisation of countries remain too simplistic. ${ }^{27}$ Health policies are better addressed by a deeper understanding of the distribution of disease burden within rather than between populations. ${ }^{28}$ The traditional dichotomy between developed and developing countries masks heterogeneity within countries and may have played an additional part in the lack of satisfactory information on this subject in Lebanon and other countries at varying stages of the epidemiological transition. In these countries, priorities in health policies should be directed towards improving reporting systems of routine sources of data, while concurrently improvising alternate strategies for collecting empirical epidemiological data and other health related indicators for advocacy and evidence-based decision making. Our results apply only to middle aged and older populations and further studies are required to determine levels and patterns of mortality and risk factors in younger age groups and in the rural populations.

We are grateful to Dr Alex Kalache for his advice early in the study and Dr Samer Jabbour for his comments on an earlier version of the paper.

Funding: this study was funded by MEAwards (MEA-290, Population Council Research Grant, West Asia and North Africa, Egypt) and is in part based on AM Sibai's PhD dissertaAfrica, Egypt) and is
tion at the LSHTM.

Conflicts of interest: none.

1 Omran AR. The epidemiologic transition. A theory of the epidemiology of population change. Milbank Memorial Fund $Q 1971 ; 49: 509-38$.

2 Dodu SRA. Emergence of cardiovascular diseases in developing countries. Cardiology 1988;75:56-64.

3 Anonymous. The hidden epidemic of cardiovascular diseases. (Editorial). Lancet 1998;352:1795.

4 Frenk J, Bobadilla LJ, Stern C, et al. Elements for a theory of the health transition. Health Transition Review 1991;1: 21-38. 
5 Murray CJL, Lopez AD. Mortality by cause for eight regions of the world: Global Burden of Disease Study. Lancet 1997; of the world:

6 United Nations Fund for Population Activities and the Ministry of Social Affairs. Statistical tables for the Population and Housing Census, 1994-1996. Beirut: MOSA, 1997.

7 Zurayk HC, Armenian HK. Beirut 1984: a population and health profile. Beirut: American University of Beirut, 1985.

8 Dora C. Risk factors for chronic disease mortality in old age: a longitudinal study in Porto Alegre, Brazil. (PhD Thesis). London: University of London, 1992.

9 Clayton D, Hills M. Analysis of follow-up studies. College Station, TX: Stata Technical Bulletin, 1995:19-26.

10 StataCorp. Stata statistical software: release 4.0 College Station, TX: Stata Corporation, USA, 1995.

11 Fletcher A. The epidemiology of cardiovascular disease in women. Vascular Med Rev 1995;313:491-8.

12 World Health Organization. Epidemiology and prevention of cardiovascular diseases in elderly people. Report of a WHO cardiovascular diseases in elderly people. Report of a WHO study group.

13 Wingard DL, Cohn BA, Kaplan G, et al. Sex differentials in morbidity and mortality risks examined by age and cause in the same cohort. Am f Epidemiol 1989;130:601-10.

14 Chandramohan D, Maude GH, Rodrigues LC, et al. Verbal autopsies for adult deaths: issues in their development and validation. Int $\mathcal{F}$ Epidemiol 1994;23:213-22.

15 Tollman SM, Kahn K, Garenne M, et al. Reversal in mortality trends: evidence from the Agincourt field site, South Africa, 1992-1995. AIDS 1999;13:1091-7.

16 Kakrani VA, Pratinidhi AK, Gupte AM. A study of registration of deaths at primary health centre with special reference to "verbal autopsy" method. Indian $7 \mathrm{Med} S \mathrm{Si}$ 1996;50:196-200.
17 Khoury SA, Massad D, Fardous T. Mortality and causes of death in Jordan 1995-96: assessment by verbal autopsy. death in Jordan 1995-96: assessment by
Bull World Health Organ 1999;7:641-50.

18 Quigley MA, Chandramohan D, Rodrigues LC. Diagnostic accuracy of physician review, expert algorithms and data-derived algorithms in adult verbal autopsies. Int $\mathcal{F}$ Epidemiol 1999;28:1081-7.

19 Abou-Daoud KT. Mortality and cause of death in the city of Beirut. Leb Med $\mathcal{f}$ 1967;20:273-89.

20 Murray CJL, Lopez AD. Global and regional cause-of-death patterns in 1990. Bull World Health Org 1994;72:447-80.

21 Abou-Daoud KT. Coronary heart disease: associations observed in hospitalised patients. Mortality and cause death in the city of Beirut. Leb Med F 1968;21:49-57.

22 Thom TJ. Stroke mortality trends. An international 23 Tunstall-Pedoe H, Kuulasmaa K, Mähönen M, et al, for the WHO MONICA Project. Contribution of trends in survival and coronary-event rates to changes in coronary survival and coronary-event rates to changes in coronary MONICA Project populations. Lancet 1999;353:1547-57.

24 Hunink MG, Goldman L, Tosteson AN, et al. The recent Hunink MG, Goldman L, Tosteson AN, et al. The recent
decline in mortality from coronary heart disease, 1980decline in mortality from coronary heart disease, 1980ment. FAMA 1997;277:535-42.

25 Gwatkin DR, Guillot M, Heuveline P. The burden of disease among the global poor. Lancet 1999;354:586-9.

26 Reddy KS. The burden of disease among the global poor. (Letter). Lancet 1999;354:1477.

27 Beaglehole R, Bonita R. Public health at the crossroads. Achievements and prospects. Cambridge: Cambridge University Press, 1999

28 Murray CJL, Lopez AD. Global burden of disease. (Author's reply). Lancet 1999;350:144-5. 